\title{
Switch Based Opportunistic Spectrum Access for General Primary User Traffic Model
}

\author{
Fakhreddine Gaaloul, Hong-Chuan Yang, Redha M. Radaydeh, and Mohamed-Slim Alouini
}

\begin{abstract}
This letter investigates the performance of a cognitive radio transceiver that can opportunistically use the available channel. We propose and compare two different opportunistic channel access schemes. The first scheme applies when the secondary user (SU) has access to only one channel. The second scheme is based on switching between available channels and applies when the SU has access to multiple channels but can at a given time monitor and access only one channel. For these proposed access schemes, we derive analytical expressions of the novel metrics of the average access duration and the average waiting time for a general primary user traffic model. Based on these two metrics a time-average secondary access throughput formula is presented to predict the performance of the $\mathrm{SU}$.
\end{abstract}

\section{INTRODUCTION}

Cognitive radio has been shown to be one of the potential solutions to radio spectrum resource scarcity [1]. The opportunistic spectrum access (OSA) techniques can achieve high spectrum efficiency and quality of service [2]-[5].

In OSA, one of the important issue is the modeling of the behavior of the PU. The PUs activity is modeled as a Markov chain with two states: "busy" and "idle" which have an associated random variable (RV) describing the amount of time spent by the PU in each state. In the case of light tailed (LT) traffic, the PU activity can be modeled by exponential distribution. This model is not always realistic, but some experimental studies have shown that it can be a reasonable approximation of the PU behavior in some systems such as the IEEE 802.11 Wireless LAN and $2 \mathrm{G}$ voice-oriented cellular systems for various traffic models [6]. Besides, empirical measurements showed in the case of data-oriented communication systems such as $3 \mathrm{G}$ cellular networks, MPEG video streams and the Internet servers traffic [7], heavy tailed (HT) traffic needs to be considered and their activities can be modeled by slowly decreasing distribution such as Pareto distribution [8].

In this paper, we propose two decentralized OSA schemes. The first one applies when the SU has access to only one channel. Thus, the SU will periodically sense the channel and access it once the PU is sensed to be OFF. The SU during transmission will sense continuously the used channel to avoid interference with PU and immediately evacuate the band as soon as the corresponding PU appears. The second proposed OSA scheme applies when the SU is able to access multiple channels but can use and sense only one channel at a time. In this case, the studied access schemes are based on two different switching schemes. The first switching scheme is the switch and examine channel access scheme (SEC), where, once the PU appears, the SU switches sequentially to the next channel and keeps switching until it find an unused channel. The second switching scheme is the switch and stay channel access scheme (SSC), in which the SU, when the PU is ON on a channel, will switch to the next channel and transmit if it is free or wait until it will be free. In this work, we introduce two novel performance metrics for SU transmission, namely average waiting duration and the average service time, which measures how much time the SU needs to wait to transmit and for how long the SU can transmit on average, respectively. We analyze the proposed OSA schemes by deriving analytical expressions of average waiting duration and average service time under different PU traffic models. These metrics are then applied to evaluate the average throughput of the secondary access. With these novel performance metrics and their accurate mathematical characterization, the type of application the secondary system can support can be predicted for different PU traffic pattern.

\section{SySTEM MODEL}

It is assumed that a PU system has $L$ parallel channels available for transmission. A cognitive SU will try to access one of the available channels opportunistically. We assume that the occupancy of each channel by the PU system evolves independently according to a homogeneous continuous-time Markov chain with idle (OFF) and busy (ON) states, with the notion that the PU traffic is not slotted. We denote the duration of the ON and OFF period of the PU by $T_{o n}^{p}$ and $T_{o f f}^{p}$, respectively. The SU transmitter, is assumed to always have data to send, to have unslotted traffic protocol and to have the ability to sense and access only one channel at a time. Also, we assume that, when the SU is using an idle channel, it will sense continuously that channel and stop accessing it when the PU becomes ON. Thus, the proposed OSA scheme causes no interference to the PU system. We assume in this work that the channel sensing is perfect and the channel sensing results at the transmitter and receiver are the same. In this scenario, the receiver will expect to receive the signal from the transmitter when it senses the channel is free. The transmitted signal from secondary transmitter has some preambles known to the receiver to facilitate the synchronization for detection.

\section{Performance Analysis}

\section{A. Single Channel Access}

In this subsection, we assume that the $\mathrm{SU}$ has access to only one channel. When the PU is sensed to be ON, the SU will periodically sense the channel every period of $T_{s}$. Once the SU finds that the PU is OFF, it starts transmitting while sensing the PU activity continuously. 


\section{1) Case of General PU Traffic:}

a) Average SU Service Time: We denote by $T_{o n}^{s}$ and $T_{o f f}^{s}$ the duration of the $\mathrm{ON}$ and OFF periods for the SU, respectively. , as shown in the block diagram in Fig. 1. During

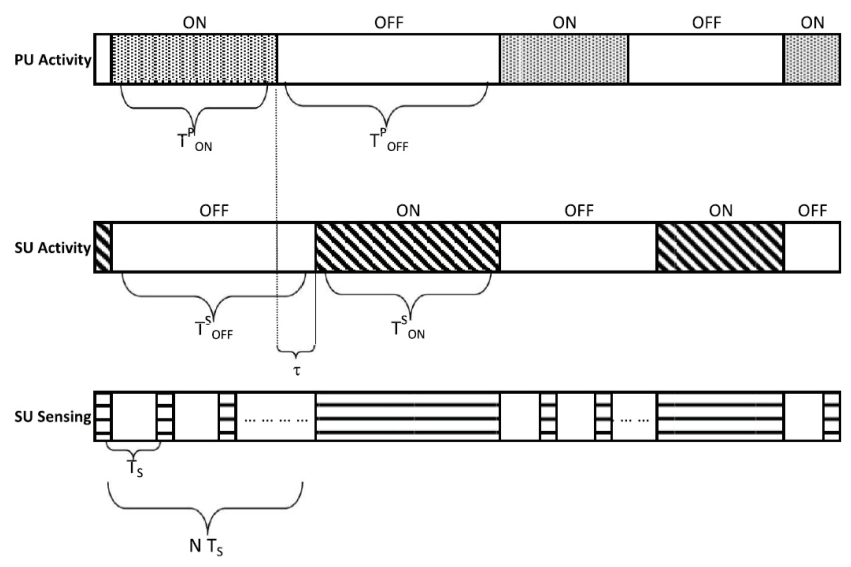

Fig. 1. Block diagram

the ON period of the PU, the SU performs a periodic sensing with period $T_{s}$. Thus the OFF duration of the SU can be expressed as $T_{o f f}^{s}=N T_{s}$, where $N$ is a $\mathrm{RV}$ that represents the number of the sensing periods before the SU switch to ON state. It is clear that $T_{o n}^{p}+T_{o f f}^{p}=T_{o n}^{s}+T_{o f f}^{s}$. Thus, the ON duration of the $\mathrm{SU}$ can then be expressed as $T_{o n}^{s}=T_{o f f}^{p}-\tau$, where $\tau=N T_{s}-T_{o n}^{p}$ represents the time duration when the $\mathrm{PU}$ is OFF and the $\mathrm{SU}$ is not transmitting, $\tau \in\left[0, T_{s}\right]$.

Conditioning on $N, T_{o n}^{p} \mid N$ is between $(N-1) T_{s}$ and $N T_{s}$. Thus, knowing the distribution of $T_{o n}^{p}$, the PDF of the RV $T_{o n}^{p} \mid N$ is a truncated PDF. For a general PU activity model, let $F_{T_{o n}^{p}}($.$) be the cumulative distribution function (CDF) of$ $T_{o n}^{p}$, the PDF of $T_{o n}^{p}$ conditioned on $N$ is given, when $N T_{s} \leq$ $t \leq(N-1) T_{s}$, by

$$
f_{T_{o n}^{p} \mid N}(t)=\frac{f_{T_{o n}^{p}}(t)}{F_{T_{o n}^{p}}\left(N T_{s}\right)-F_{T_{o n}^{p}}\left((N-1) T_{s}\right)} .
$$

Using this result, we can express the PDF of $\tau$ knowing $N$ as

$$
f_{\tau \mid N}(t)=\frac{f_{T_{o n}^{p}}\left(N T_{s}-t\right)}{F_{T_{o n}^{p}}\left(N T_{s}\right)-F_{T_{o n}^{p}}\left((N-1) T_{s}\right)}, 0 \leq t \leq T_{s} .
$$

The probability mass function (PMF) of $N$ can be expressed as a function of the CDF of $T_{o n}^{p}$ as

$$
\begin{aligned}
P_{n} & =\operatorname{Pr}\{N=n\}=\operatorname{Pr}\left\{(n-1) T_{s} \leq T_{o n}^{p} \leq n T_{s}\right\} \\
& =F_{T_{o n}^{p}}\left(n T_{s}\right)-F_{T_{o n}^{p}}\left((n-1) T_{s}\right) .
\end{aligned}
$$

Having $P_{n}$ and $f_{\tau \mid N}($.$) , it follows that$

$$
f_{\tau}(t)=\sum_{n=1}^{+\infty} f_{T_{o n}^{p}}\left(n T_{s}-t\right), 0 \leq t \leq T_{s} .
$$

Using the preceding results, the average SU service duration, for general PU traffic model, becomes

$$
\begin{aligned}
& \bar{T}_{o n}^{s}=E\left[T_{o f f}^{p}\right]-\sum_{n=1}^{+\infty} \int_{0}^{T_{s}} t f_{T_{o n}^{p}}\left(n T_{s}-t\right) d t \\
& =E\left[T_{o f f}^{p}\right]+E\left[T_{o n}^{p}\right]-\sum_{n=1}^{+\infty} n T_{s}\left(F_{T_{o n}^{p}}\left(n T_{s}\right)-F_{T_{o n}^{p}}\left((n-1) T_{s}\right)\right),
\end{aligned}
$$

where $E[$.$] is the expected value operator.$

b) Average Waiting Time: The SU waiting time, denoted by $\delta$, can be given for the case of one channel access, by $\delta=N T_{s}$. Using the PMF of $N$ in (3), it can be written that

$$
\bar{\delta}=\sum_{n=1}^{+\infty} n T_{s}\left[F_{T_{o n}^{p}}\left(n T_{s}\right)-F_{T_{o n}^{p}}\left((n-1) T_{s}\right)\right] .
$$

2) Application to LT Traffic: Generally speaking, a RV is LT if its distribution decrease exponentially or faster such as exponential, Gamma, and Weibull with shape parameter larger than one [8]. An example of LT traffic is the Poisson traffic where the duration of the ON and OFF period are exponentially distributed and their PDFs are given by

$$
f_{T_{o n}^{p}}(t)=\frac{1}{\lambda} e^{-\frac{t}{\lambda}} U(t), f_{T_{o f f}^{p}}(t)=\frac{1}{\mu} e^{-\frac{t}{\mu}} U(t),
$$

where $\lambda$ and $\mu$ represent the average duration of the ON period and the OFF period, respectively, and $U($.$) is the unit step$ function. Based on the results in the previous subsection for general PU traffic and using the statistics of the PU activity given in (7), the average $\mathrm{SU}$ access duration can be obtained as $\bar{T}_{o n}^{s}=\mu+\lambda-\frac{T_{s}}{1-e^{-\frac{T_{s}}{\lambda}}}$. In the case of Poisson PU traffic, the PMF of $N$ defined in (3) can be expressed as $P_{n}=e^{-(n-1) \frac{T_{s}}{\lambda}}\left(1-e^{-\frac{T_{s}}{\lambda}}\right)$. Thus $\bar{\delta}$ is given by

$$
\bar{\delta}=\frac{T_{s}}{1-e^{-\frac{T_{s}}{\lambda}}} .
$$

It can be seen that when $T_{s} \rightarrow+\infty$, which means that the SU continuously senses the channel, $\frac{T_{s}}{1-e^{-\frac{T_{s}}{\lambda}}} \rightarrow \lambda$. So $\bar{T}_{o n}^{s}$ is approaching $\mu$ and the waiting time for the $\mathrm{SU}$ is approaching $\lambda$, as expected, because in this case the SU will begin transmitting exactly when the PU finish and will finish exactly when the PU begin transmitting.

3) Application to HT Traffic: Generally speaking, a RV is HT if its distribution decrease slower than exponentially. Some typical examples are Pareto, log-normal, and Weibull with shape parameter larger than one [8]. The heavy tailed PU traffic has different behavior compared to the LT traffic. Specifically, the HT PU busy state can have infinite mean and variance which can considerably degrade the SU performance and reduce significantly the network throughput by increasing the waiting time of the SU.

In this paper, we take, as an example of the HT traffic, the exponential distribution for the OFF duration of the PU with mean $\mu$ and Pareto distribution for the $\mathrm{ON}$ duration of the PU with shape parameter $\alpha>1$ and scale parameter $x_{m}>0$. 
Thus, the distribution of the ON and OFF duration of the PU activity are given by

$$
f_{T_{o n}^{p}}(x)=\frac{\alpha x_{m}^{\alpha}}{x^{\alpha+1}} U\left(x-x_{m}\right), f_{T_{o f f}^{p}}(x)=\frac{1}{\mu} e^{-\frac{x}{\mu}} U(x) .
$$

Using the distributions given in (9) and knowing that $E\left[T_{o n}^{p}\right]=\frac{\alpha x_{m}}{\alpha-1}, \alpha>1$ and $E\left[T_{o f f}^{p}\right]=\mu$, we can get the average service and waiting duration of the SU defined for general PU traffic model in (5) and (6), respectively, and express them, when $n_{0} T_{s}<x_{m} \leq\left(n_{0}+1\right) T_{s}$, as

$\bar{T}_{o n}^{s}=\mu+\frac{\alpha x_{m}}{\alpha-1}-\bar{\delta}, \bar{\delta}=\left(n_{0}+1\right) T_{s}+\frac{x_{m}^{\alpha}}{T_{s}^{\alpha-1}} \zeta\left(\alpha, n_{0}+1\right)$,

where $\zeta(s, q)$ is the Hurwitz zeta function.

\section{B. Multi-Channel Access Based on SEC Scheme}

In this section, we assume that the SU can access any one of all the available channels. If the sensed channel is idle, the SU will use it and sense it continuously until the PU is present. Once the PU is present in the channel, the SU will switch to the next channel. We will assume that when the SU switches to a new channel, the switching duration is a part of the sensing duration $T_{p}$.

1) Case of General PU Traffic:

a) Average SU Service Time: The service time for the $\mathrm{SU}$, for the considered scheme, can be given by $T_{o n}^{s}=T_{o f f}^{p}-$ $W-T_{p}$, where $W$ is a uniform RV defined on the interval $\left[0, T_{o f f}^{p}-T_{p}\right]$ and its PDF is given by

$$
f_{W}(t)=\frac{1}{T_{o f f}^{p}-T_{p}}\left(U(t)-U\left(t-T_{o f f}^{p}+T_{p}\right)\right) .
$$

The average SU transmission time can be given by

$$
\begin{aligned}
\bar{T}_{o n}^{s} & =E\left[T_{o f f}^{p}\right]-\int_{0}^{\infty} E\left[W / T_{o f f}^{p}\right] f_{T_{o f f}^{p}}(t) d t-T_{p} \\
& =\frac{1}{2}\left(E\left[T_{o f f}^{p}\right]-T_{p}\right) .
\end{aligned}
$$

b) Average Waiting Time: Given the channel and sensing time $T_{p}$, the waiting time is a multiple of $T_{p}$ and can be given by $\delta=N T_{p}$, where $N$ is a $\mathrm{RV}$ that represents the number of channels that the SU has to examine before finding a free channel. The discrete RV $N$ can be modeled by a Bernoulli RV with probability $p=\frac{E\left[T_{o n}^{p}\right]}{E\left[T_{o n}^{p}\right]+E\left[T_{o f f}^{p}\right]}$. Therefore, the PMF of $N$ is

$$
P_{n}=E\left[T_{o f f}^{p}\right] E\left[T_{o n}^{p}\right]^{n-1}\left(E\left[T_{o n}^{p}\right]+E\left[T_{o f f}^{p}\right]\right)^{-n} .
$$

Finally, we can calculate and express $\bar{\delta}$ as

$$
\bar{\delta}=\sum_{n=1}^{+\infty} n T_{p} P_{n}=\frac{T_{p}}{E\left[T_{o f f}^{p}\right]}\left(E\left[T_{o n}^{p}\right]+E\left[T_{o f f}^{p}\right]\right) .
$$

2) Application to LT Traffic: For a LT PU traffic, the average SU service time is given by $\bar{T}_{o n}^{s}=\frac{1}{2}\left(\mu-T_{p}\right)$, and the average waiting duration, given in (14) for general case, is expressed as $\bar{\delta}=\frac{T_{p}}{\mu}(\lambda+\mu)$.
3) Application to HT Traffic: For the SEC scheme when considering a HT traffic, the average service duration of the $\mathrm{SU}$ is the same as in the case of the LT case and is given by $\bar{T}_{o n}^{s}=\frac{1}{2}\left(\mu-T_{p}\right)$. Using (14),we can get the SU waiting time and express it as $\bar{\delta}=\frac{T_{p}}{\mu}\left(\mu+\frac{\alpha x_{m}}{\alpha-1}\right)$.

\section{Multi-Channel Access Based on SSC Scheme}

In this section, we assume that the SU has access to all the available channels and sense the channels sequentially. If the sensed channel is idle, the SU transmits and senses the channel continuously until the PU is present. Once the PU is present in the channel, the SU switches to the next channel. If the next channel is available, the SU can transmit. Otherwise, it stays on that channel and wait until it becomes free. During the waiting time, the SU senses periodically (every $T_{s}$ ) the PU activity. In this part, we denote by $T_{p}$ and $T_{s}$ the duration of sensing and the period of sensing, respectively. Note that the switching duration is assumed to be negligible and the waiting is exactly the same as the case of single channel access given for general PU traffic in (6) and given for LT and HT traffics in (8) and (10), respectively. In the next subsection we will focus only on the SU service duration.

1) Case of General PU Traffic: To calculate the average service time for this switching scheme, we need to consider two cases, which are i) the case when the SU switches to another channel and find it idle. We denote the service duration in this case by $T_{1}$. ii) the case when the switch to channel is found to be busy. We denote the service duration in this case by $T_{2}$. The service time is in these cases is given by $T_{o n}^{s}=p_{1} T_{1}+p_{2} T_{2}$, where $p_{1}$ and $p_{2}$ are the probability to find the channel idle and busy, respectively, and are given by $p_{1}=\frac{E\left[T_{o f f}^{p}\right]}{E\left[T_{o n}^{p}\right]+E\left[T_{o f f}^{p}\right]}$ and $p_{2}=\frac{E\left[T_{o n}^{p}\right]}{E\left[T_{o n}^{p}\right]+E\left[T_{\text {off }}^{p}\right]}$.

When the switch to channel is found to be idle, the SU waits for $T_{p}$ duration to switch and to sense the channel and then transmit. Thus in this case knowing that we found the switch to channel idle, the average service time will be exactly the same as of the case of SEC scheme, given by $\bar{T}_{1}=\frac{1}{2}\left(E\left[T_{o f f}^{p}\right]-T_{p}\right)$. When the switch-to channel is found to be busy, the SU will stay in that channel and wait until the channel becomes free. During the waiting time, the SU will sense the channel periodically every $T_{s}$. Thus the service time in this case is the same as in one channel case as given in (5)

2) Application to LT Traffic: For Poisson PU traffic, using the general formulations in the previous part, the average SU service duration can be given by

$$
\bar{T}_{o n}^{s}=\lambda+\frac{1}{2} \frac{\mu\left(\mu-T_{p}\right)}{\mu+\lambda}-\frac{1}{\mu+\lambda}\left(\frac{T_{s}}{1-e^{-\frac{T_{s}}{\lambda}}}\right) .
$$

3) Application to HT Traffic: For HT traffic, the average SU service time can be written as

$$
\begin{aligned}
& \bar{T}_{o n}^{s}=\frac{\alpha x_{m}}{\alpha-1}+\frac{1}{2} \frac{\mu(\alpha-1)\left(\mu-T_{p}\right)}{\alpha x_{m}+\mu(\alpha-1)}-\frac{\alpha x_{m}}{\alpha x_{m}+\mu(\alpha-1)} \\
& \times\left[\left(n_{0}+1\right) T_{s}+\frac{x_{m}^{\alpha}}{T_{s}^{\alpha-1}} \zeta\left(\alpha, n_{0}+1\right)\right] .
\end{aligned}
$$




\section{Time-Average Secondary Access Throughput}

In this part, we present a performance metric, namely time-average secondary access throughput, based on the two previously proposed metrics, average service time and average waiting time. The average channel throughput for the considered access scheme is given by

$$
\overline{\mathcal{T}}=\frac{\bar{T}_{o n}^{s}}{\bar{\delta}+\bar{T}_{\text {on }}^{s}} \int_{0}^{\infty} \log _{2}(1+\gamma) p_{\gamma}(\gamma) d \gamma,
$$

where $\gamma$ is the signal-to-noise ratio of the $\mathrm{SU}, p_{\gamma}(\gamma)$ is the PDF of the received SNR of the SU, and $\bar{T}_{o n}^{s}$ and $\bar{\delta}$ are defined above for different schemes and traffic models.

\section{Numerical Results AND Discussions}

Fig. 2, shows the average service duration of the SU for the different studied access schemes and for the LT traffic model as a function of the average OFF duration of the PU $\mu$. It is clear that as $\mu$ increases the service duration for the SU and for all the considered schemes increases and this is because the OFF period of the PU will be longer. On the other hand, it can be seen that the one channel access scheme have better service duration compared to switching based schemes. Fig. 3, shows

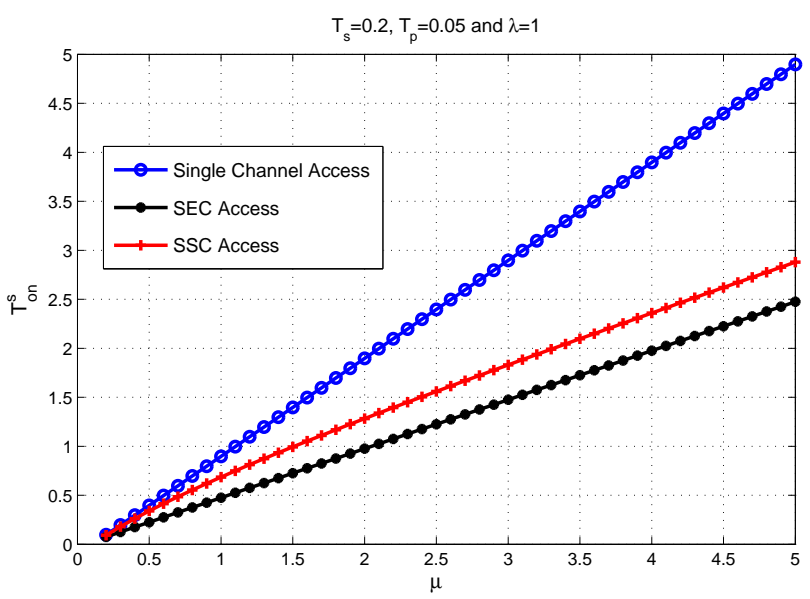

Fig. 2. Average SU service duration for the different proposed schemes and for LT traffic model.

the average waiting time of the SU for the different studied access schemes and for the HT traffic model as a function of $x_{m}$. It is clear that as $x_{m}$ increases the SU waiting duration for all the considered schemes increases and this is because the ON period of the PU will be longer. On the other hand, the trade of between the access and waiting durations can be seen from both the figures, since the schemes presenting the highest access duration have also the highest waiting duration. Besides, the advantage of using the SEC scheme is clear as it can reduce the waiting time substantially.

\section{CONCLUSION}

This paper has investigated different single channel and multiple channels OSA schemes. The multiple channels access schemes are based on two different switching strategy namely

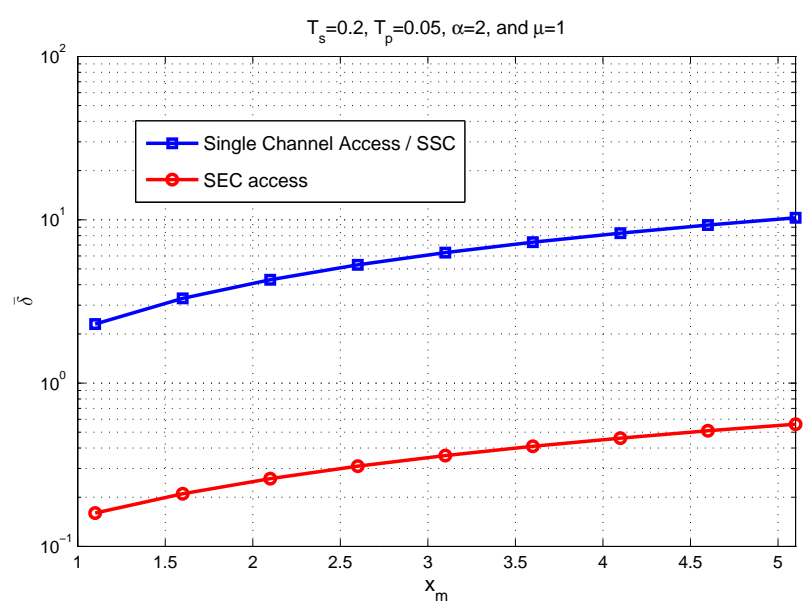

Fig. 3. Average SU waiting duration for the different proposed schemes and for HT traffic model.

the SEC and SSC. The average waiting and service time for SU are presented and derived for the proposed schemes and for general PU traffic model to predict the performance of the SU. Then, these metrics are obtained in closed-form for two different applications of PU traffic model which are the HT and LT traffics. Through the results, the performance of each access scheme has been thoroughly studied. Numerical results showed that single channel access scheme presents the highest average service time, however the SEC scheme presents the lowest waiting duration for the considered sensing strategy.

\section{REFERENCES}

[1] S. Haykin, "Cognitive radio: brain-empowered wireless communications," IEEE Journal on Selected Areas in Communications, vol.23, no.2, pp. 201- 220, Feb. 2005.

[2] M. H. Islam, C. L. Koh, S. W. Oh, Xianming Qing, Y. Y. Lai, Cavin Wang, Ying-Chang Liang, B. E. Toh, F. Chin, G. L. Tan, and W. Toh, "Spectrum Survey in Singapore: Occupancy Measurements and Analyses," 3rd International Conference on Cognitive Radio Oriented Wireless Networks and Communications, 2008, (CrownCom 2008), pp.17, 15-17 May 2008.

[3] B. Hamdaoui, "Adaptive spectrum assessment for opportunistic access in cognitive radio networks," IEEE Transactions on Wireless Communications, vol.8, no.2, pp.922-930, Feb. 2009.

[4] Z. Qianchuan, S. Geirhofer, T. Lang and B.M. Sadler, "Opportunistic Spectrum Access via Periodic Channel Sensing," IEEE Transactions on Signal Processing, vol.56, no.2, pp.785-796, Feb. 2008.

[5] Z. Qing, T. Lang, S. Ananthram and C. Yunxia, "Decentralized cognitive MAC for opportunistic spectrum access in ad hoc networks: A POMDP framework," IEEE Journal on Selected Areas in Communications, vol.25, no.3, pp.589-600, April 2007.

[6] S. Geirhofer, L. Tong, and B. M. Sadler, "Dynamic spectrum access in WLAN channels: Empirical model and its stochastic analysis," presented at the 1st Int. Workshop Technol. Policy Accessing Spectrum (TAPAS), Boston, MA, Aug. 2006.

[7] M. Wellens and P. Mahonen, "Lessons Learned from an Extensive Spectrum Occupancy Measurement Campaign and a Stochastic Duty Cycle Model," J. Mobile Networks and Applications, vol. 15, no. 3, pp. 461-474, 2010

[8] P. Wang, I. F. Akyildiz, "On the Origins of Heavy-Tailed Delay in Dynamic Spectrum Access Networks," IEEE Transactions on Mobile Computing, vol.11, no.2, pp.204-217, Feb. 2012. 